\title{
Dynamics near the surface reconstruction of W(100)
}

\author{
M. R. Baldan \\ Laboratório Associado de Sensores e Materiais, Instituto Nacional de Pesquisas Espaciais, 12201-190 São José dos Campos, \\ São Paulo, Brazil \\ and Faculdade de Engenharia, Universidade São Francisco, Itatiba, São Paulo, Brazil \\ E. Granato \\ Laboratório Associado de Sensores e Materiais, Instituto Nacional de Pesquisas Espaciais, 12201-190 São José dos Campos, \\ São Paulo, Brazil \\ S. C. Ying \\ Department of Physics, Brown University, Providence, Rhode Island 02912
}

(Received 23 November 1999)

\begin{abstract}
Using Brownian molecular dynamics simulation, we study the surface dynamics near the reconstruction transition of W(100) via a model Hamiltonian. Results for the softening and broadening of the surface phonon spectrum near the transition are compared with previous calculations and with He atom scattering data. From the critical behavior of the central peak in the dynamical structure factor, we also estimate the exponent of the power-law anomaly for adatom diffusion near the transition temperature.
\end{abstract}

The $(1 \times 1)$ to $c(2 \times 2)$ structural transition on $\mathrm{W}(100)$ has been one of the best characterized and studied surface phase transitions. ${ }^{1,2}$ Besides the obvious interest in surface science as an important surface structural transition, it provides a physical realization of the universality class of the two-dimensional $X Y$ model with fourfold symmetry breaking fields. ${ }^{3,4}$ Moreover, the strength of the symmetry breaking field can be tuned by varying the coverage of adsorbates such as hydrogen atoms. In particular, the field can be made to vanish at a particular coverage to realize the pure twodimensional $X Y$ model. $^{4}$ As such, the study and understanding of this transition has significance far beyond this system itself. It is of importance in the general field of statics and dynamics of two-dimensional phase transitions. The equilibrium properties of the $\mathrm{W}(100)$ transition are well understood through extensive experimental and theoretical investigations. On the other hand, the critical dynamics for this transition has received relatively little attention. Experimentally, there exists only one study that probes the softening and damping of the phonon modes ${ }^{1}$ near the critical temperature $T_{c}$. However, the details of the critical dynamics such as the emergence of the central peak and the dynamical critical exponents have not been investigated. Previous theoretical studies of the dynamics for this transition consist of a finite slab geometry molecular dynamics (MD) simulation study ${ }^{5}$ and an analytic continued fraction (CF) expansion method. ${ }^{6}$ Both of these approaches have limitations near $T_{c}$ either because of the small size limit in MD or because of the truncation of the CF expansion after a few steps. In particular, although the softening and overdamping of the vibrational modes were confirmed in these studies, both these studies were not able to determine the dynamical and static critical exponents that characterize the critical region quantitatively.

Recently, there are renewed interests in studying the critical dynamics of the W(100) surface reconstruction using different experimental probes. One approach that has been proposed is a time resolved laser and low-energy electron diffraction (LEED) spectroscopy, which consists of an initial laser pulse that heats up the system followed by synchronized electron beam pulse that probes the subsequent temporal variation of the order parameter for the $c(2 \times 2)$ phase. $^{2}$ This is a difficult experiment and has not been attempted yet. More recently, Xiao and Altman ${ }^{7}$ propose to probe directly the temperature dependence of the diffusion constant of adsorbed atoms on W(100) using the degradation of the diffraction signal from a laser-written grating on the adlayer. The interest in this study arises from the theoretical prediction that the adatom diffusion constant would vanish at the transition due to the coupling to the critical fluctuations in the substrate. ${ }^{8}$ This experiment is under way and the results should yield better understanding of critical fluctuations near $T_{c}$ for this surface.

In view of the ongoing experimental study and the inadequacies of the previous theoretical investigations, we have undertaken a study of the surface dynamics near the W(100) reconstruction through the direct simulation of the Langevin dynamics of a two-dimensional effective Hamiltonian, which has been very successful in the study of the equilibrium properties of this transition. ${ }^{9}$ The approximate $\mathrm{CF}$ study of the dynamics is also based on this Hamiltonian. ${ }^{6}$ Unlike the previous MD studies, the simplicity of this effective Hamiltonian allows us to use much larger sizes, which is important for probing critical fluctuation effects. We also avoid the truncation approximations used in the analytic continued fraction study. ${ }^{6}$ The only drawback of using a twodimensional effective Hamiltonian is that the longwavelength phonons, which penetrate deeply into the bulk of the substrate, cannot be described accurately within this approach. However, unlike the excitations near the critical wave vector $q_{0}=(\pi / a, \pi / a)$, these long-wavelength phonons are scarcely affected by the critical fluctuations and contributes little to the strong temperature dependence of dynamical quantities near $T_{c}$. As shown below, with the larger size of the present study, we are able to collapse all 
our data for the dynamical structure factor based on a general dynamical scaling analysis. This allows us to extract the static and dynamical critical exponents, as well as extending the validity of the results arbitrarily close to $T_{c}$.

The clean $\mathrm{W}(100)$ surface is described by an effective Hamiltonian in dimensionless form ${ }^{9}$

$$
\begin{aligned}
H= & \sum_{i}\left\{\frac{p_{i}^{2}}{2}+\frac{A}{2} u_{i}^{2}+\frac{B}{4} u_{i}^{4}+8 H_{4} u_{i x}^{2} u_{i y}^{2}+C_{1}\left(u_{i} \cdot u_{j}+u_{i} \cdot u_{k}\right)\right. \\
& \left.+C_{2}\left[u_{i x}\left(u_{j^{\prime} y}-u_{k^{\prime} y}\right)+u_{i y}\left(u_{j^{\prime} x}-u_{k^{\prime} x}\right)\right]\right\},
\end{aligned}
$$

where $p_{i}$ is the momentum vector and $u_{i}$ the displacement vector of a W atom from the $i$ th site, $R_{i}=a_{0}(l, m)$, with $l$ and $m$ integers, and $j=(l+1, m), k=(l, m+1), j^{\prime}=(l+1, m$ $+1)$, and $k^{\prime}=(l-1, m+1)$. The dimensionless parameters used in the simulation are the same as for model $\mathrm{I}$ in the previous study: ${ }^{9} A=-10, B=40, C_{1}=3.75, C_{2}=C_{1} / 2$, and $H_{4}=-1.85$. For this choice of parameters, the ground state displacement is $u_{0}=1$, and the transition temperature has been determined previously from Monte Carlo simulations ${ }^{9}$ to be $T_{c}=2.11$. The scale factors of distance $L_{s}$ and temperature $T_{s}$ are then chosen so that $u_{0}$ and $T_{c}$ agree with the experimentally observed values, $0.2 \AA$ and $230 \mathrm{~K}$, respectively, and have the values $L_{s}=0.2 \AA$ and $T_{s}=109 \mathrm{~K}$. This effective Hamiltonian has been very successful in accounting for the static critical phenomena such as the detailed temperature dependence and anisotropy of the intensity of the LEED diffraction pattern ${ }^{9}$ near $T_{c}$. This Hamiltonian belongs to the universality class of the $X Y$ model with the fourfold symmetry breaking field represented by the $H_{4}$ coupling term in the Hamiltonian. ${ }^{3,10,4}$ Thus, the static critical exponents are nonuniversal, depending on the value of the marginal field $H_{4}$. In the large coupling limit, it should correspond to the Ising universality class when $C_{2}=0$. It can also be related, by renormalization-group arguments, to the Ashkin-Teller model, which consists of two coupled Ising models and is known to have a critical line with varying static critical exponents. ${ }^{11}$ However, less is known about the dynamical critical behavior. It is not known for example whether the dynamical critical exponent $z$ is dependent on the value of the fourfold symmetry breaking field $H_{4}$. The same question arises for the critical line of the Ashkin-Teller model. ${ }^{12}$ The time scale $t_{s}$ for this model Hamiltonian is not adjustable and is determined by the relation $t_{s}$ $=\left(m_{s} L_{s}^{2} / k_{B} T_{s}\right)^{1 / 2}=0.28 \mathrm{ps}$, where $m_{s}$ is the $\mathrm{W}$ atom mass. In the previous CF study, ${ }^{6}$ it was demonstrated that the softening and damping of the surface phonons observed in the He scattering experiment ${ }^{1}$ are well described quantitatively without any other adjustable parameters by the results of this model up to the point where the soft modes become completely overdamped.

To simulate the dynamical behavior of the system described by the Hamiltonian in Eq. (1) we use standard methods of Brownian molecular dynamics. ${ }^{13}$ The equations of motion for the particle coordinates are given by

$$
m \frac{d^{2} u_{i}}{d t^{2}}+m \eta_{0} \frac{d u_{i}}{d t}=-\frac{\partial H}{\partial u_{i}}+f_{i},
$$

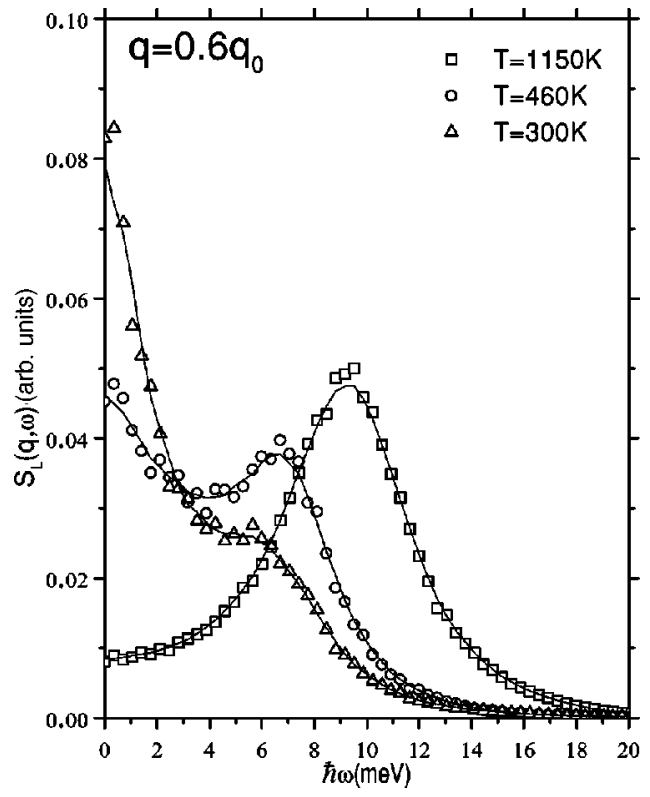

FIG. 1. Longitudinal structure factor $S_{L}(\mathbf{q}, \omega)$ for $\mathbf{q}=0.6 \mathbf{q}_{0}$ at different temperatures.

where $f$ is a random force with each component $\alpha$ satisfying

$$
\left\langle f_{i \alpha}(t) f_{j \beta}(0)\right\rangle=2 m k_{B} T \eta_{0} \delta(t) \delta_{i j} \delta_{\alpha \beta},
$$

and $\eta_{0}$ is a microscopic damping parameter representing the frictional coupling of the effective two-dimensional system to the heat bath consisting of the other subsurface layers. A $N \times N$ square lattice with periodic boundary conditions has been used in the simulations. Most calculations were done with $N=32$ and $N=46$. The equations of motion (2) are integrated numerically with a time step of $\delta t=0.01 t_{s}$ in reduced units of time $t_{s}$. The damping coefficient parameter $\eta_{0}$ cannot be determined from equilibrium quantities like the other parameters in the effective Hamiltonian. To determine $\eta_{0}$, we compare the width of the phonon modes calculated from the present model in a regime far from $T_{c}$ with the experimental data. ${ }^{1}$ For $\eta_{0} \geqslant 0.05$, the noncritical phonon modes have widths substantially larger than the observed experimental value. This sets an upper bound for $\eta_{0}$. In our present investigation, we set $\eta_{0}=0.05$. Near the transition, the critical dynamics and damping are dominated by the interparticle interactions and the precise value of $\eta_{0}$ is immaterial.

The dynamic properties of the system can be most conveniently studied by examining the dynamic structure factor $S_{\alpha \beta}(\mathbf{q}, \omega)$ defined as

$$
S_{\alpha \beta}(\mathbf{q}, \omega)=\int e^{i \omega t}\left\langle u_{\alpha}^{*}(\mathbf{q}, t) u_{\beta}(\mathbf{q})\right\rangle d t,
$$

where $\alpha$ and $\beta$ represent the components of the displacement. We consider in this paper mainly the longitudinal $S_{L}(q, \omega)$ and transverse $S_{T}(q, \omega)$ components of the tensor $S_{\alpha \beta}(\mathbf{q}, \omega)$, obtained from displacements $u_{\alpha}$ along the direction of the vector $\mathbf{q}$ and perpendicular to $\mathbf{q}$, respectively. For a given wave vector $\mathbf{q}$, the longitudinal [transverse] phonon mode shows up as a peak in $S_{L}(\mathbf{q}, \omega)\left[S_{T}(\mathbf{q}, \omega)\right]$, while the width of the peak corresponds to the damping of the mode. In Figs. 1, 2, 3, and 4, the behavior of the calculated dynamic 


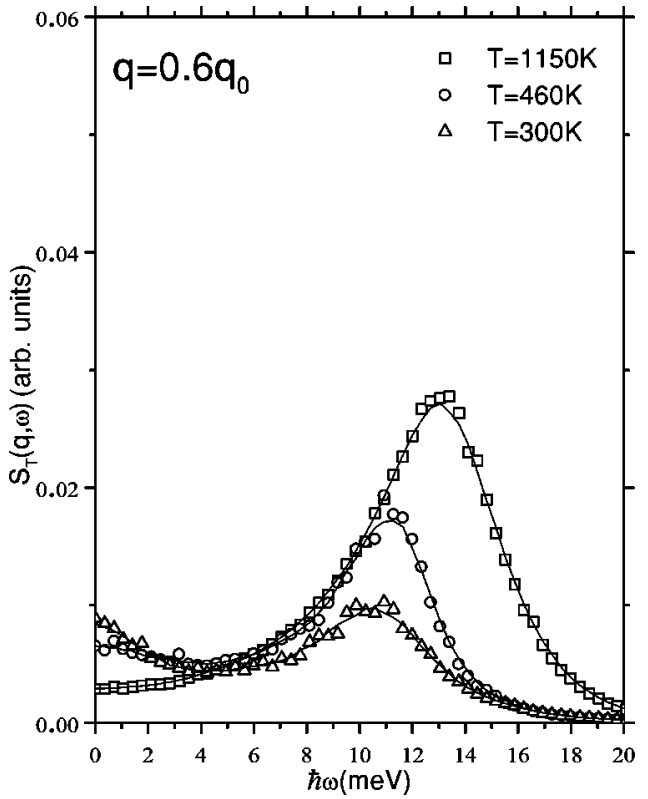

FIG. 2. Transverse structure factor $S_{T}(\mathbf{q}, \omega)$ for $\mathbf{q}=0.6 \mathbf{q}_{0}$ at different temperatures.

structure factor as a function of temperature near the critical temperature $T_{c} \sim 230 \mathrm{~K}$ are shown. As can be seen from the figures, as one approaches $T_{c}$ from above, both the longitudinal and transverse modes near $\mathbf{q}_{0}=(\pi / a, \pi / a)$ start to soften and broaden until they are completely overdamped at $T \sim 1.5 T_{c}$. As one further approaches $T_{c}$, the dynamics is completely dominated by a strong central peak. These results are qualitatively similar to that obtained in the previous study through the analytic $\mathrm{CF}$ expansion approach. ${ }^{6}$ Moreover, the peak position and width of the soft phonon modes in the two approaches are approximately the same as shown in Figs. 5 and 6 . Thus, the good agreement between the theoretical results for the soft modes and the experimental $\mathrm{He}$ atom scattering data as demonstrated previously ${ }^{6}$ remains true.

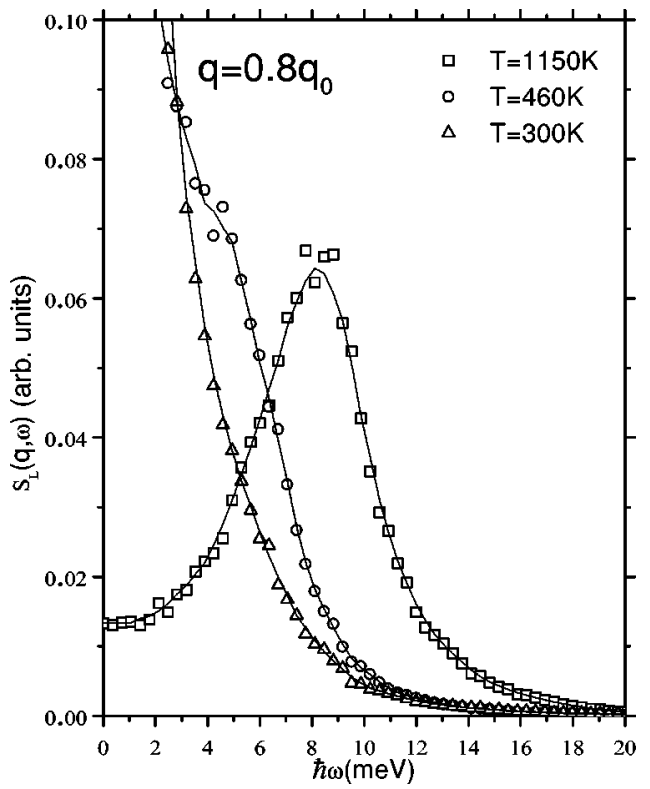

FIG. 3. Longitudinal structure factor $S_{L}(\mathbf{q}, \omega)$ for $\mathbf{q}=0.8 \mathbf{q}_{0}$ at different temperatures.

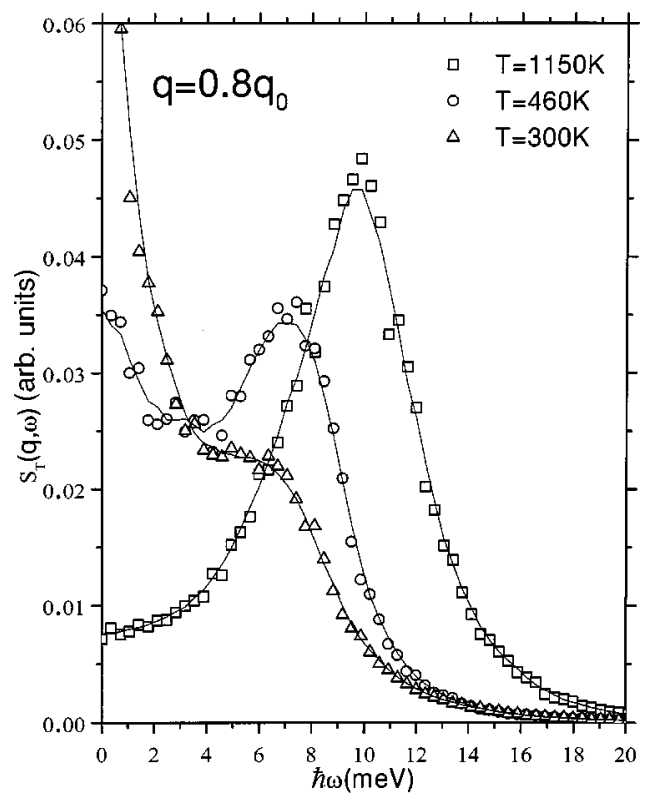

FIG. 4. Transverse structure factor $S_{T}(\mathbf{q}, \omega)$ for $\mathbf{q}=0.8 \mathbf{q}_{0}$ at different temperatures.

However, as shown in Fig. 6, the present results show a stronger central peak relative to the soft phonon mode near the critical point when compared with the CF calculation. ${ }^{6}$ This is due to the fact that a truncation of the $\mathrm{CF}$ after a few steps introduces effectively a finite size limitation, leading to an underestimate of the critical fluctuation effects. This difference in the relative strength of the central peak could be important in such physical quantities as the anomalous temperature dependence of the diffusion constant as discussed below. It also shows that the apparent convergence of the $\mathrm{CF}$ approach after a few steps can be deceptive, and the quantitative behavior of the critical dynamics needs a long expansion of the $\mathrm{CF}$, which may render the analytical approach impractical.

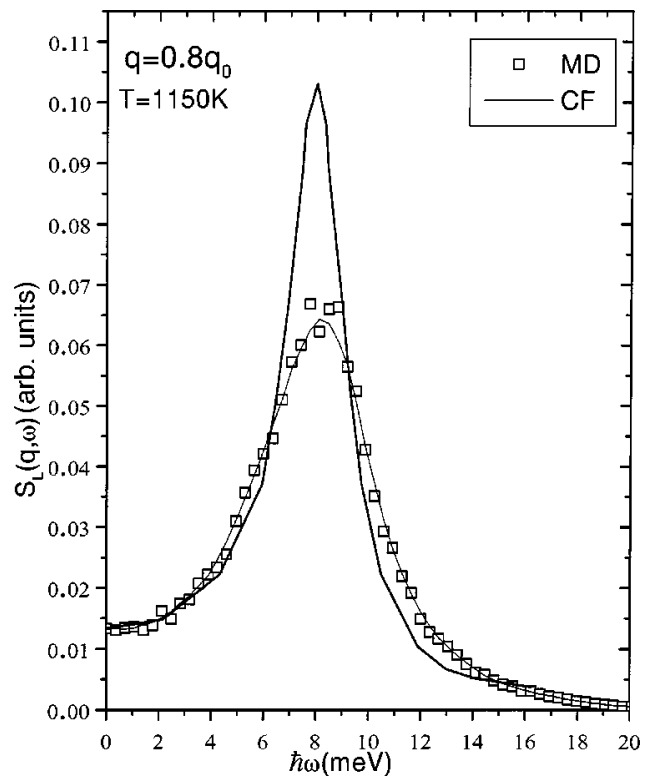

FIG. 5. Longitudinal structure factor $S_{L}(\mathbf{q}, \omega)$ far from $T_{c}$ for $\mathbf{q}=0.8 \mathbf{q}_{0}$ as obtained from the present study and the CF approximation (Ref. 6). 


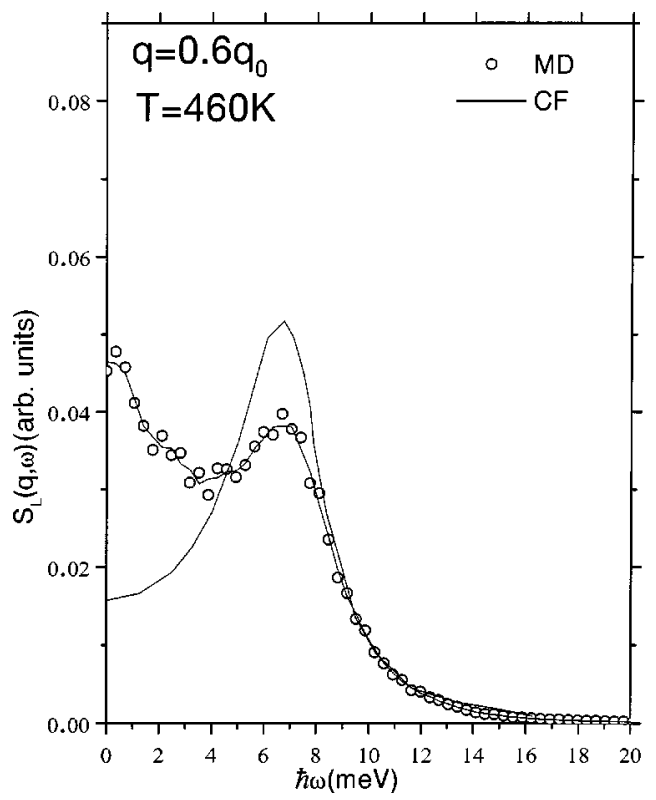

FIG. 6. Longitudinal structure factor $S_{L}(\mathbf{q}, \omega)$ close to $T_{c}$ for $\mathbf{q}=0.6 \mathbf{q}_{0}$ as obtained from the present study and the CF approximation (Ref. 6).

We now examine the critical properties more closely by focusing on the central peak. It follows from standard scaling arguments that near $T_{c}$, the dynamical structure factor should satisfy the scaling form

$$
N^{d} S(\tilde{q}, \omega)=\xi^{z+\gamma / \nu} g_{ \pm}\left(\tilde{q} \xi, \omega \xi^{z}\right),
$$

where $\tilde{q}=\left|\mathbf{q}-\mathbf{q}_{0}\right|, g_{ \pm}(\omega)$ is a scaling function, $\xi \propto \mid T / T_{c}$ $-\left.1\right|^{-\nu}$ is the divergent correlation length and $d$ is the system dimension. We now focus on $S(\omega) \equiv S(\tilde{q}=0, \omega)$. The results for $S(\omega)$ from the present calculation for various $\omega$ and $\mathrm{T}$ near $T_{c}$ are plotted in Fig. 7. Note that at $\mathbf{q}=\mathbf{q}_{0}$ or $\tilde{q}=0$, the longitudinal and transverse modes are identical and $S(\omega)$ is

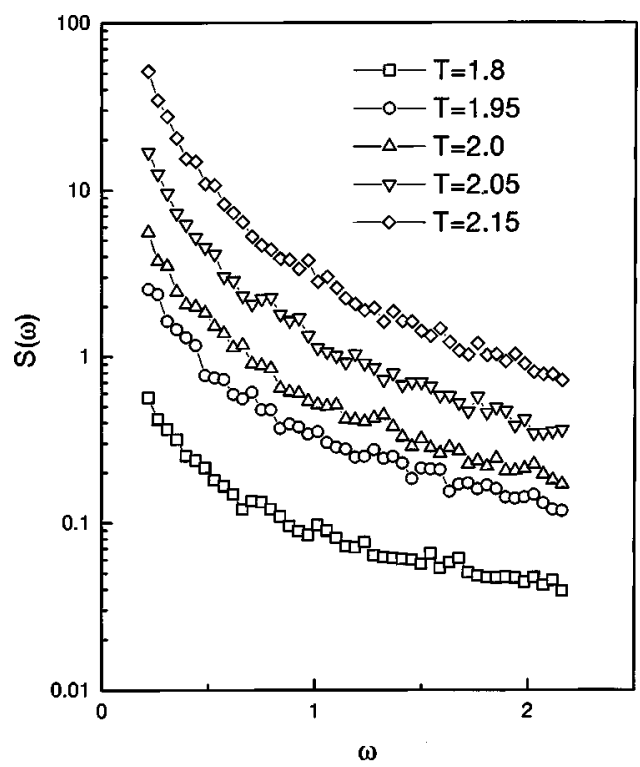

FIG. 7. Structure factor $S(\omega)$ at $\mathbf{q}=\mathbf{q}_{0}$ as a function of dimensionless temperature $T$ and frequency $\omega$ near $T_{c}$. The units for $T$ and $\omega$ are $T_{s}$ and $t_{s}^{-1}$, as described in the text.

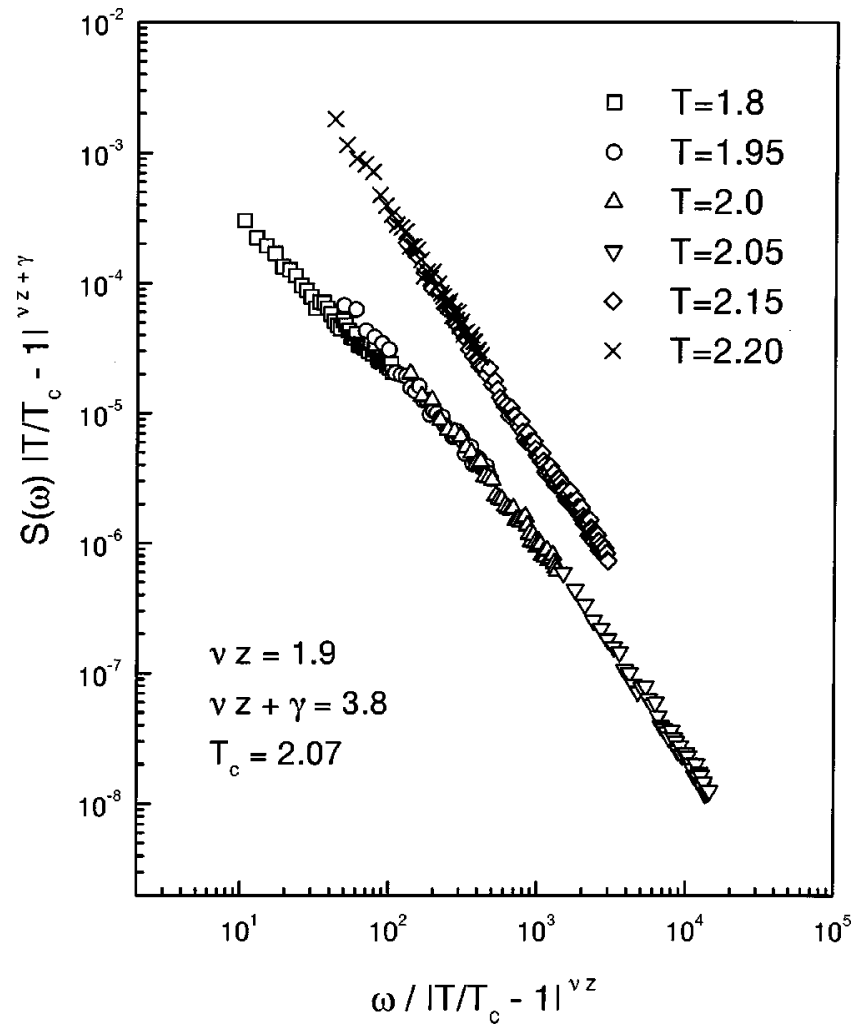

FIG. 8. Scaling plot of $S(\omega)\left|T / T_{c}-1\right|^{\nu z+\gamma} \omega\left|T / T_{c}-1\right|^{-\nu z}$ from the data in Fig. 7 obtained by adjusting the parameters $T_{c}, \nu z$ and $\nu z+\gamma$. The upper set of collapsing data corresponds to $T>T_{c}$ and the lower one to $T<T_{c}$.

isotropic. According to the scaling form in Eq. (4), the data in a scaling plot $S(\omega)\left|T / T_{c}-1\right|^{\nu z+\gamma} \omega\left|T / T_{c}-1\right|^{-z \nu}$ should collapse on to a single curve above or below $T_{c}$, respectively, if the exponents $\nu z+\gamma$ and $z \nu$ and the critical temperature $T_{c}$ are chosen correctly. This scaling plot is shown in Fig. 8 for a system size $N=32$. By adjusting the parameters for the exponents and $T_{c}$, we have verified that the data for $S(\omega)$ indeed obey the scaling hypothesis. We obtain the values $T_{c}=2.07(4), \nu z=1.9(4)$, and $\nu z+\gamma=3.8(4)$. The same analysis was performed for a larger system size $N$ $=46$ with the same results, indicating that finite-size corrections are negligible in the range of frequency and temperatures studied. The resulting value of $\gamma=1.9(4)$ is consistent with previous estimate from equilibrium simulations that found $^{9} \gamma=1.5(2)$ and $\nu=0.83(1)$. Combining this value of $\nu$ with the present results from dynamic scaling, we get an estimate for the dynamic critical exponent $z=2.3(4)$. It is interesting to note that according to the dynamical scaling arguments, $S(\omega=0)$ diverges as $\left|T / T_{c}-1\right|^{-(\nu z+\gamma)}$ as one approaches $T_{c}$. In the analytic CF approach however, a truncation of the continued fraction for $S(\omega=0)$ at the second stage yields an approximate divergent behavior of $\mid T / T_{c}$ $-\left.1\right|^{-2 \gamma}$ for $S(\omega=0)$. For the present model, these two exponents $\nu z+\gamma$ and $2 \gamma$ are indistinguishable within our numerical error. Another interesting observation is that the value of the dynamical critical exponent $z$ determined here is close to the corresponding value for the Ising model, which lies in the range 2.08 to 2.24 as obtained by numerical and renormalization-group calculations. ${ }^{14}$ It raises the interesting question of whether the dynamic exponent can be universal 
even when the static critical exponents are known to be dependent on the value of the marginal field $H_{4}$. Further work on dynamics of the $X Y$ model with fourfold symmetry breaking using more efficient Monte Carlo methods for larger size systems are under way. ${ }^{15}$ They should help to clarify more the issue of dynamic universality class for this model. However, for the related Ashkin-Teller model, recent numerical results ${ }^{12}$ show that the dynamical exponent $z$ does not vary significantly along the nonuniversal critical line compared with the error estimates and is also close to the results for the Ising model. Thus, we expect that the present estimate of $z$ may be a good approximation for our model with a weak dependence on the parameters of the model.

While the soft modes and the central peak can be directly probed experimentally with $\mathrm{He}$ scattering studies, ${ }^{1}$ it also influences indirectly physical quantities such as the adatom diffusion constant, which depends strongly on the coupling of the adatom to the substrate excitations. As shown previously, ${ }^{8}$ the friction $\eta$ acting on the adatom due to the coupling to the substrate phonon excitations is related to the zero-frequency limit of the dynamical structure factor,

$$
\eta \propto \int d^{2} \tilde{q} S(\tilde{q}, \omega=0)|W(\tilde{q})|^{2},
$$

where $W(\tilde{q})$ denotes the Fourier coefficients of the coupling of the adatom to the substrate. Thus, adatom diffusion measurements ${ }^{7}$ will probe the dynamic correlation function $S(\tilde{q}, \omega=0)$ which diverges as the temperature approaches $T_{c}$ because of the central peak excitations. According to the general scaling relation given in Eq. (4), the friction coefficient $\eta$ should diverge near the transition as $\left.\mid T / T_{c}-1\right]^{-x}$, with the critical exponent ${ }^{16} x=\nu(z-2)+\gamma$. The difference of the exponent for $\eta$ from that of $S(\omega=0)$ arises from the integration over the wave vectors for the friction coefficient. Since $D \propto 1 / \eta$, this leads to the prediction of a vanishing diffusion constant as one approaches $T_{c}$, with a power-law anomaly $D \propto\left|T / T_{c}-1\right|^{x}$. The divergent behavior of the friction parameter near $T_{c}$ is not just specific to this system. Diffusion anomalies near a surface preroughening transition has also been predicted ${ }^{17}$ based on similar considerations. Finally, we have shown earlier ${ }^{18}$ that the effective pinning strength of flux lattice in disordered type II superconductors should diverge at the flux-lattice melting transition due to the coupling of the pinning center to the critical fluctuations. This could be the possible origin for the "peak effect" observed in high- $T_{c}$ superconductors in a magnetic field. Recent experimental data ${ }^{19}$ show a precise correlation of the peak effect with the vortex lattice melting transition, lending support to this picture.

In conclusion, we have performed a Langevin simulation study of the dynamics of the W(100) surface reconstruction near the transition point. We are able to avoid the severe size limitations of the previous molecular dynamical studies by studying an effective two-dimensional Hamiltonian and analyze the results through dynamical scaling theory. We have determined both the static and dynamical critical exponents for this transition, which belongs to the universality class of the $X Y$ model with fourfold anisotropy. Finally, we have related the central peak of the phonon spectrum to the friction experienced by an adatom on this surface. The adatom diffusion constant on this surface is predicted to vanish at the transition point due to the divergent frictional damping. The comparison of our new results with forthcoming adatom diffusion data would provide a detailed picture and further understanding of the critical dynamics near this intriguing surface reconstruction. Furthermore, the divergent nature of the friction near the transition is not just specific to this system but should manifest itself near other continuous phase transitions such as the surface preroughening transition and the vortex lattice melting transition in type-II superconductors.

This work was supported in part by a joint NSF-CNPq grant and by FAPESP Grant No. 99/02532-0 (E.G.) and 98/ 00977-2 (M.R.B.).
${ }^{1}$ H.-J. Ernst, E. Hulpke, and J.P. Toennies, Phys. Rev. B 46, 16 081 (1992).

${ }^{2}$ J.R. Thompson, P.M. Weber, and P.J. Estrup, in Time-Resolved Electron and Diffraction, edited by P.M. Rentzeps (SPIE, San Diego, 1995), p. 113.

${ }^{3}$ J.V. José, L.P. Kadanoff, S. Kirkpatrick, and D.R. Nelson, Phys. Rev. B 16, 1217 (1977).

${ }^{4}$ T. Ala-Nissila, E. Granato, K. Kankaala, J.M. Kosterlitz, and S.C. Ying, Phys. Rev. B 50, 12692 (1994).

${ }^{5}$ C.Z. Wang, A. Fasolino, and E. Tosatti, Phys. Rev. B 37, 2116 (1988).

${ }^{6}$ W.H. Han, S.C. Ying, and D. Sahu, Phys. Rev. B 41, 4403 (1990).

7 X.D. Xiao and M. Altman (private communication).

${ }^{8}$ T. Ala-Nissila, W.K. Han, and S.C. Ying, Phys. Rev. Lett. 68, 1866 (1992).

${ }^{9}$ W.K. Han and S.C. Ying, Phys. Rev. B 41, 4403 (1990).

${ }^{10}$ G.Y. Hu and S.C. Ying, Physica A 140A, 585 (1987).

${ }^{11}$ B. Nienhuis, in Phase Transitions and Critical Phenomena, edited by C. Domb and J.L. Lebowitz (Academic Press, London,
1987), Vol. 11, p. 1.

${ }^{12}$ Z.B. Li, X.W. Liu, L. Schülke, and B. Zheng, Physica A 245, 485 (1997).

${ }^{13}$ M.P. Allen and D.J. Tildesley, Computer Simulation of Liquids (Oxford University Press, Oxford, 1987).

${ }^{14}$ V.V. Prudnikov, A.V. Ivanov, and A.A. Fedorenko, Pis'ma Zh. Éksp. Teor. Fiz. 66, 793 (1997) [JETP Lett. 66, 835 (1997)]

${ }^{15}$ E. Falck, T. Ala-Nissila, S. C. Ying, and E. Granato (unpublished).

${ }^{16}$ The expression for the critical exponent $x$ obtained here from dynamical scaling is more general than the one obtained previously (Ref. 8) based on an approximate relation between $S(q, \omega)$ and the displacement susceptibility.

${ }^{17}$ P. Prestipino, G. Santoro, and E. Tosatti, Phys. Rev. Lett. 75, 4468 (1995).

${ }^{18}$ T. Ala-Nissila, E. Granato, and S.C. Ying, J. Phys.: Condens. Matter 2, 8537 (1990).

${ }^{19}$ J. Shi, X.S. Ling, R. Liang, D.A. Bonn, and W.N. Hardy, Phys. Rev. B 60, R12 593 (1999). 